\title{
Does auto-PAP work in patients with acute quadriplegia and sleep- disordered breathing?
}

\author{
Abdulghani Sankari, ${ }^{1,2}$ M. Safwan Badr ${ }^{1,2}$
}

Patients living with spinal cord injury or disease (SCI/D) face numerous hurdles to accessing high-quality care for sleep disorders, from inadequate recognition of sleep health among patients with SCI/D and their providers, to erroneous attribution of daytime symptoms, limited access to sleep diagnostic services and difficulties using medical devices needed to treat sleep-disordered breathing (SDB). The treatment of choice for SDB is positive airway pressure (PAP), which improves sleep quality, metabolic function and quality of life while reducing sleepiness in able-bodied adults. For example, the Apnea Positive Pressure Long-term Efficacy Study (APPLES) trial, ${ }^{1}$ which recruited from both sleep clinics and the general population, demonstrated that PAP therapy was associated with significant improvement in subjectively and objectively measured sleepiness, but without a significant impact on short-term or long-term cognitive function. Even in able-bodied adults with SDB, adherence to PAP remains a major challenge that requires individualised and intensive education, monitoring and support. There is evidence that patients with SCI/D are less adherent to PAP therapy than ablebodied individuals. ${ }^{2}$ Reasons for poor adherence include mobility impairment to adjust the mask, increased awakenings due to autonomic dysfunction, nasal congestion, inadequate support and inconvenience due to the presence of other comorbidities. $^{3}$

SDB in patients with SCI/D combines features of obstructive and central SDB. For example, increased upper airway collapsibility is noted in all patients with $\mathrm{SCI}^{4}$ and the use of opioid analgesics may increase the risk of central SDB. In addition, patients with chronic cervical SCI demonstrate increased propensity

\section{${ }^{1}$ Division of Pulmonary, Critical Care and Sleep Medicine, Department of Internal Medicine, Wayne State University School of Medicine, Detroit, Michigan, USA \\ ${ }^{2}$ Department of Internal Medicine, John D Dingell VAMC, Detroit, Michigan, USA}

Correspondence to Dr Abdulghani Sankari, Wayne State University School of Medicine, Detroit, MI 48201, USA; asankari@wayne.edu to central apnoea, ${ }^{5}$ marked sleep-onset hypoventilation $^{6}$ and enhanced chemoreflex sensitivity. ${ }^{7}$ These physiologic derangements may necessitate individualised management approaches. Access to diagnostic sleep laboratories for patients with limited mobility is another significant challenge. Consequently, many patients with SCI are evaluated using home sleep apnoea testing, followed by auto-PAP (APAP). However, the validity of this approach has not heretofore been validated.

In this issue of the journal, Berlowitz and colleagues ${ }^{8}$ published the first randomised controlled trial testing the effect of APAP use over 3 months in patients with acute cervical SCI. The primary outcome was attention and information processing using Paced Auditory Serial Addition Task (PASAT) tools. The participants were randomised to APAP $(n=73)$ or no treatment $(n=76)$. The major findings were that the use of APAP therapy in patients with acute traumatic cervical SCI during inpatient rehabilitation was associated with improved daytime sleepiness but not neurocognitive function as no significant difference was found in attention or information processing at the end of the 3-month study period. These data are generally consistent with findings in able-bodied individuals as shown in the APPLES trial, ${ }^{1}$ which showed that the use of PAP improved sleepiness but did not improve long-term neurocognitive function at 6 months and resulted in only mild improvement in the measures of executive and frontal lobe function for those with severe SDB.

Studies that demonstrate negative results are critical to optimising care by allowing scientists to focus on more promising approaches. However, accepting negative findings to guide future care requires methodological rigour and clarity regarding the population of interest. Accordingly, the results of the study are not ipso facto sufficient to conclude that PAP is not an effective therapy for SDB in this population. The main limitation is the low adherence rate of $21 \%$ and average use of 2.9 hours/night. It is of note that the sample size was based on an adherence rate of $85 \%$. Therefore, negative findings are difficult to interpret in an underpowered study. Conversely, significant improvement in sleepiness was noted, despite the low adherence rate. Thus, it is plausible that different domains may have a different PAP use threshold as has been demonstrated by Weaver et al in ablebodied patients with sleep apnoea. ${ }^{9}$

Several factors may also affect the interpretation and generalisability of the findings. First, medications, such as Baclofen, may have an independent effect on neurocognition, ${ }^{10}$ potentially masking a beneficial effect of PAP therapy. Second, the inclusion of participants during the acute phase only may be non-representative of the population of chronic cervical SCI. In fact, Berlowitz et al have previously shown that the prevalence of SDB decreases after the initial acute peak. ${ }^{11}$ Therefore, it is possible that SDB, and its consequences, may spontaneously resolve in some patients regardless of the treatment arm. Third, the efficacy of APAP in patients with acute cervical SCI who are prone to recurrent central apnoea and marked nocturnal hypoventilation remains uncertain. Finally, excluding participants with hypercapnia and those who were intolerant of PAP may have eliminated a potentially responsive subset of patients.

Several broad conclusions can be drawn from the findings of the study. The improvement in daytime sleepiness indicates the effectiveness of PAP for sleepy patients with cervical SCI and SDB. In addition, the feasibility of providing APAP without in-laboratory testing may enhance acceptance and potentially adherence rate. Conversely, the observation of low APAP adherence supports the need to develop and test patient-centred interventions to enhance acceptance, usability and adherence, including referral to a sleep specialist, which may be associated with higher PAP adherence rates. ${ }^{12}$

The pathophysiologic complexity of SDB in patients with cervical SCI may influence the outcome of PAP therapy. We posit that narrowing the use of APAP to those with obstructive SDB may enhance the efficacy of APAP therapy. Therefore, we propose a structured approach for management of SDB in patients with SCI based on the underlying type of SDB and associated hypoxia or hypoventilation (figure 1).

Berlowitz et al are to be congratulated on the rigour and transparency of their carefully conducted study. This work also underscores the unique challenges facing scientists conducting clinical trials in this population. While the negative results for 


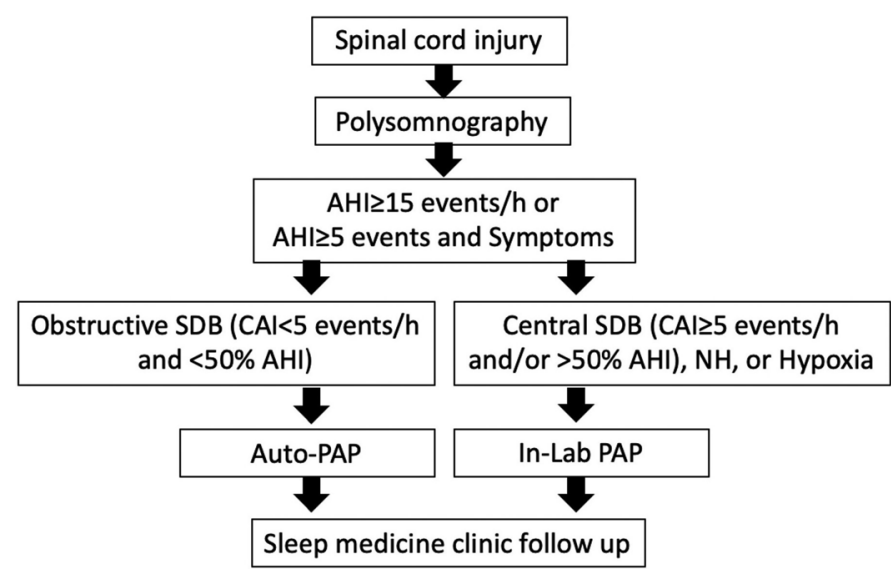

Figure 1 Proposed management pathway for SDB in patients with spinal cord injury (SCI). AHI, apnoea-hypopnoea index; CAl, Central apnea index; NH, nocturnal hypoventilation; SDB, sleepdisordered breathing.

the primary outcome are disappointing, the results in aggregate will inform future research on the treatment of SDB in patients with cervical SCI. The magnitude of benefit cannot be inferred from the study, owing to the low adherence rate. However, the improvement in daytime sleepiness supports the use of PAP in this population. Whether APAP is the optimal choice in patients with SCI/D remains unclear, given the propensity to develop central apnoea. Finally, there is a clear need to pursue investigating trait-specific therapies for SDB and testing methods to enhance acceptance and adherence to PAP.

Contributors Both authors contributed to drafting and approving the final version of this editorial.

Funding Dr Sankari is supported by the United States VA Office of Research and Development, Department of Veterans Affairs No. 1IK2CX000547, and RX002885 and the National Heart, Lung, and Blood Institute Award No. R21HL140447. Dr Badr is supported by the VA Office of Research and Development, Department of
Veterans Affairs No. 1101RX002116, the National Heart, Lung, and Blood Institute Award No. R01HL130552 and the Department of Defense No. SC150201.

Disclaimer This was not an industry-supported study. The opinions expressed in this article reflect those of the authors and do not necessarily represent the official views of the US Government, the Department of Veterans Affairs or the National Institute of Health.

Competing interests None declared.

Patient consent Not required.

Provenance and peer review Commissioned; internally peer reviewed.

(C) Author(s) (or their employer(s)) 2019. No commercial re-use. See rights and permissions. Published by BMJ.

\section{Check for updates}

To cite Sankari A, Badr MS. Thorax 2019;74:217-218.

Accepted 10 December 2018

Published Online First 4 January 2019

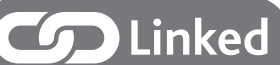

- http://dx.doi.org/10.1136/thoraxjnl-2018-212319

Thorax 2019;74:217-218.

doi:10.1136/thoraxjnl-2018-212729

\section{REFERENCES}

1 Kushida CA, Nichols DA, Holmes TH, et al. Effects of continuous positive airway pressure on neurocognitive function in obstructive sleep apnea patients: The Apnea Positive Pressure Long-term Efficacy Study (APPLES). Sleep 2012;35:1593-602.

2 Sankari A, Martin JL, Bascom AT, et al. Identification and treatment of sleep-disordered breathing in chronic spinal cord injury. Spinal Cord 2015;53:145-9.

3 Sankari A, Martin JL, Badr MS. Sleep disordered breathing and spinal cord injury: Challenges and opportunities. Curr Sleep Med Rep 2017;3:272-8.

4 Sankari A, Bascom A, Badr M. Upper airway mechanics in chronic spinal cord injury during sleep (vol 116, pg 1390, 2014). Journal Of Applied Physiology 2014;117:939.

5 Sankari A, Bascom AT, Chowdhuri S, et al. Tetraplegia is a risk factor for central sleep apnea. J Appl Physiol 2014;116:345-53. 1985

6 Bascom AT, Sankari A, Goshgarian HG, et al. Sleep onset hypoventilation in chronic spinal cord injury. Physiol Rep 2015;3:e12490.

7 Bascom AT, Sankari A, Badr MS. Spinal cord injury is associated with enhanced peripheral chemoreflex sensitivity. Physiol Rep 2016;4:e12948.

8 Berlowitz DJ, Schembri R, Graco M, et al. COSAQ Collaborative. Positive airway pressure for sleepdisordered breathing in acute quadriplegia: a randomised controlled trial. Thorax 2019;74:282-90.

9 Weaver TE, Maislin G, Dinges DF, et al. Relationship between hours of CPAP use and achieving normal levels of sleepiness and daily functioning. Sleep 2007;30:711-9.

10 Stackman RW, Walsh TJ. Baclofen produces doserelated working memory impairments after intraseptal injection. Behav Neural Biol 1994;61:181-5.

11 Berlowitz DJ, Brown DJ, Campbell DA, et al. A Iongitudinal evaluation of sleep and breathing in the first year after cervical spinal cord injury. Arch Phys Med Rehabil 2005;86:1193-9.

12 Gordon A, Wu SJ, Munns N, et al. Untreated sleep apnea: An analysis of administrative data to identify risk factors for early nonadherence. J Clin Sleep Med 2018;14:1303-13. 